\title{
Prueba sensorial de preferencia para dos dip tipo hummus a base de Gandul (Cajanus cajan (L.) Mill sp.) con sabor a alcachofa y aceituna
}

Recibido: 20 Enero, 2013

\author{
Carmen Andrés Jiménez' \& Karol Saravia Zúñiga \\ 1. UNED.candres@uned.ac.cr \\ 2. UNED. ksaravia@uned.ac.cr
}

\section{RESUMEN}

En la zona de San Carlos se cuenta con un total de 12 productores que están cultivando Gandul (Cajanus cajan) y entre las variedades más importantes que se cultivan se encuentra la Criolla, Enana, Catie y Panameña. Actualmente se está buscando darle un valor agregado al grano como fuente de ingreso alternativa para los productores. Por medio de una prueba de preferencia que establece los gustos del consumidor ante un producto innovador, nuevo o con un valor agregado, se estudiaron dos presentaciones diferentes de dip tipo hummus a base de gandul criollo: uno a base de aceituna y otro a base de alcaparras. La prueba se aplicó a un total de 39 panelistas y por medio de la tabla de significancia para pruebas de dos muestras no se encontraron diferencias significativas $(\mathrm{p}>0.05)$. Se recomienda realizar un panel de grado de satisfacción para cada uno de los dip tipo hummus con un panel de al menos 40 panelistas no entrenados para obtener un resultado más objetivo de su aceptación, además de realizar un focus group sobre los mismos productos para definir con más precisión su posible lanzamiento al mercado.

Palabras clave: Gandul, prueba de preferencia, consumidor, hummus, aceituna, alcaparra.

\footnotetext{
ABSTRACT

San Carlos is a place where 12 farmers are planting Gandul (Cajanus cajan). Among the most important varieties grown are: Folk, Catie, Enana and Panameña. Finding ways to add value to this leguminous is a current interest to offer the producers an alternative income source. Through a preference test, which establishes the tastes of the consumer for a new product, two different presentations of hummus type dip made from Gandul were studied: one made with olive and the other with caper. The test was applied to 39 people and through a two sample test significance table no significant differences were found among the samples ( $\mathrm{p}$ $>0.05)$. A satisfaction sensorial test can be done for each product to determine their acceptance in a more objective way with at least 40 panelists. Also, a focus group could
}

Aceptado: 27 Enero, 2013

be done with both products to define their possibilities in the market.

Key words: Gandul, preferente test, consummer, hummus, olive, caper.

\section{Introducción}

Según el Ministerio de Agricultura y Ganadería (1991), el gandul o frijol de palo es un arbusto perenne que crece entre 1 a $3 \mathrm{~m}$ de altura y madura en cinco meses o más. Se consume como grano tierno (arveja) y grano seco. También puede utilizarse como barrera viva para detener la erosión, como cultivo de sombra en los almácigos o viveros y como tapa vientos en regiones donde ocurren vientos fuertes.

El Gandul es un cultivo alternativo que no es conocido aún en Costa Rica, pero es considerado una alternativa importante para colaborar con la seguridad alimentaria, dadas sus propiedades nutricionales, ambientales y agronómicas (INTA, 2009).

Según Robledo, L (s.f), el Gandul es un cultivo de alto valor nutritivo, sus granos contienen proteínas, carbohidratos, vitaminas y minerales. Además, según González (2012), este cultivo enriquece los suelos por brindar nutrientes y nitrógeno al suelo, además de proteger las cuencas hidrográficas.

La Asociación de Agricultores y Productores de Gandul, Raíces y Tubérculos ha estado cultivando el Gandul desde el año 2010 gracias al proyecto establecido por la UNED en conjunto con el ICE, en la zona de La Tigra y Chachagua de San Carlos. 
Esta asociación ha tenido dificultades para surgir económicamente a partir de sus propios cultivos (raíces y tubérculos), por lo que se decidió introducir 8 variedades de Gandul para generarles un ingreso extra.

Las variedades más importantes de este cultivo son: Criolla, Catie, Panameña y Enana. También se ha comprobado que otra variedad conocida como Sabalito soporta las inclemencias del tiempo y da muy buena cosecha (Céspedes, 2012).

Aunado a lo anterior, además de su siembra, se implementó una estrategia para desarrollar subproductos derivados del gandul (Cajanus cajan (L) Millsp.) que cuenten con alto valor agregado y nutricional y para esto se decidió realizar pruebas de análisis sensorial.

El objetivo de esta investigación fue aplicar pruebas de preferencia para conocer si los consumidores prefieren el dip tipo hummus a base de Gandul con sabor a alcaparra o con sabor a aceituna.

En alimentos y en productos de consumo masivo existen dos enfoques principales para la evaluación sensorial con consumidores: la medida de preferencia y la de aceptación (Heymann \& Lawless, 1999, citado por Artavia, 2010, p. 18).

En las pruebas de preferencia se le presenta al panelista dos muestras codificadas y se le pide que escoja cual de las dos prefiere y para que sea más representativa se le puede pedir que exponga sus razones sobre la decisión tomada (Hernández, 2005); según esta misma autora estas pruebas se aplican en casos en los que se requiere el desarrollo de un nuevo producto.

Debido a que el dip tipo hummus elaborado a partir del Gandul criollo es uno de los productos que más aceptación ha tenido por las personas que lo han probado (Andrés, 2013-por publicarse); se ha decidido profundizar su estudio por medio de diferentes herramientas de mercadeo y científicas, como lo es, en este caso, la prueba sensorial de aceptación.

El hummus es un producto de origen árabe cuyo significado es "garbanzo" y se cree que fue utilizado por los Egipcios hace más de 7000 años y sembrado en el mediterráneo hace 5000 (Boskou, 2012). El dip tipo hummus a base de
Gandul sería un producto que competiría con el elaborado a base de garbanzo, pero se distinguiría por ser cultivado por productores nacionales y por ser un producto orgánico (González, 2012).

Entre los ingredientes que se utilizan para elaborar el hummus se encuentran la aceituna y la alcaparra y en el focus group realizado para determinar los descriptores sensoriales del dip tipo hummus a base de Gandul (Andrés, 2013-por publicar) se determinó que el sabor a aceituna y alcaparra podría ser de interés para la población.

El estudio fue realizado en las instalaciones de la Universidad Estatal a Distancia de Costa Rica, en Mercedes de Montes de Oca, con funcionarios y visitantes que llegaron de todo el país a la Feria de Investigación-Extensión 2012 organizado por la Escuela de Ciencias Exactas y Naturales de la UNED.

\section{Materiales y métodos}

La metodología utilizada para realizar esta prueba de preferencia fue la siguiente:

\section{Convocatoria de participantes}

Para realizar las pruebas de preferencia, se preparó un stand de degustación durante el Encuentro de Proyectos de InvestigaciónExtensión 2012 organizado por la Escuela de Ciencias Exactas y Naturales de la UNED.

39 funcionarios y estudiantes de la universidad participaron de esta prueba. Sin embargo se descartaron 4 de ellos por no haber llenado correctamente la hoja de respuestas.

\section{Preparación de las muestras}

Se elaboraron dos tipos de dip a base de Gandul, similares al hummus.

Se colocó un código a cada uno para evitar el sesgo en la respuesta de los participantes. El número 1 tenía sabor a alcachofa y el 2 tenía sabor a aceituna.

Los ingredientes base de ambos fueron: gandul criollo, jugo de limón, ajo, tahini (mezcla de ajonjolí tostado con agua), aceite de oliva, sal, pimienta y paprika. 


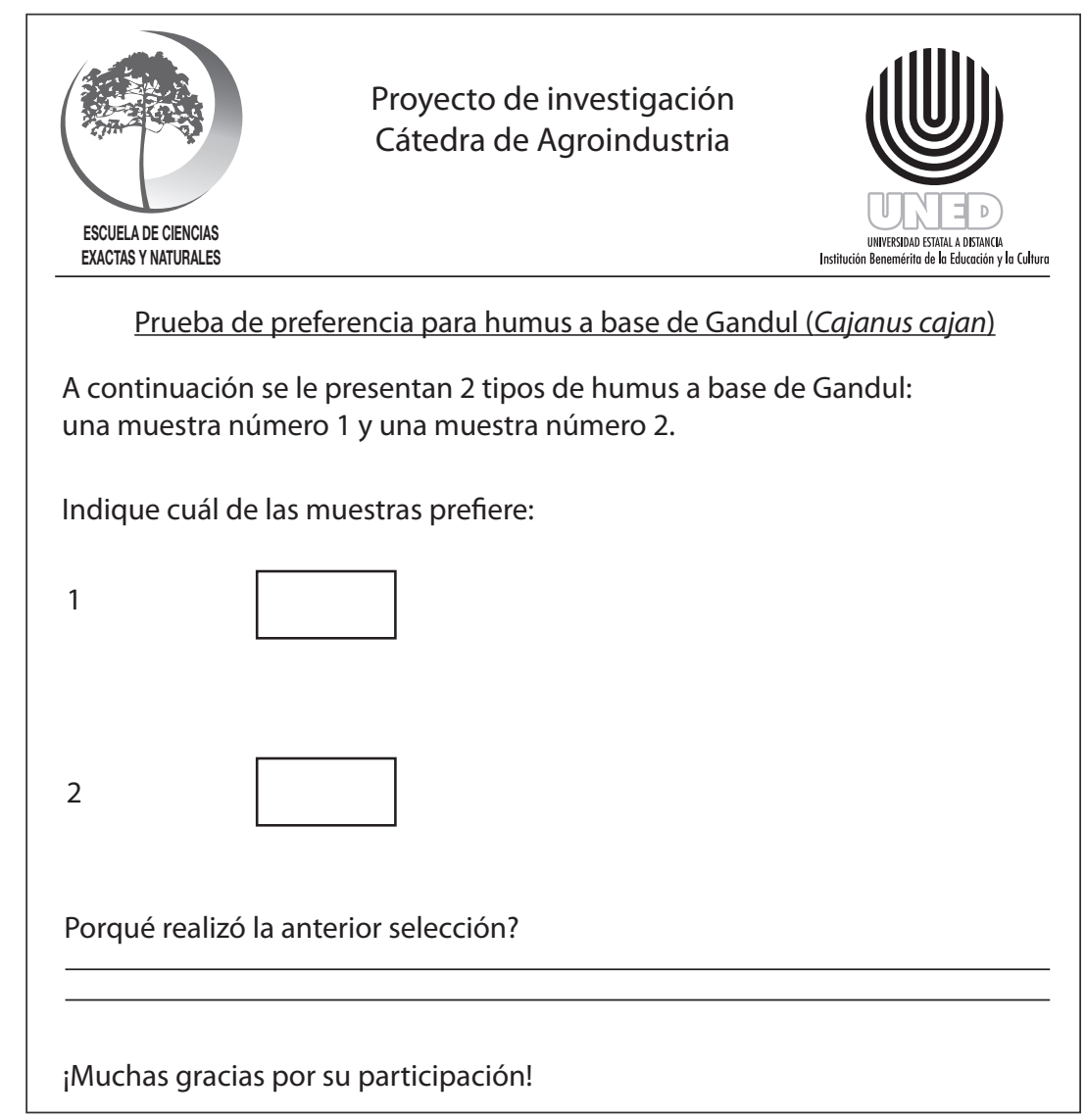

Figura 1. Formato de Prueba de Preferencia. Fuente: Autoras

A cada participante se le indicó que debía probar una porción de la muestra No. 1 para determinar su aceptación con una galleta tipo snack, posteriormente tomar agua y comer una galleta de sabor neutro.

El segundo paso consistió en probar la muestra No 2 manteniendo el mismo procedimiento que se aplicó para la muestra No 1.

Luego de realizar la degustación se le facilitó la hoja de evaluación de preferencia (figura No 1) en donde debía seleccionar la que más le gustó e indicar el porqué de la selección.

\section{Análisis estadístico}

Se utilizó la tabla de significancia para pruebas de dos muestras de Roessle et al. (1956).

Se utilizó una prueba de dos colas al 5\% de significancia, debido a que se buscó encontrar el número mínimo de juicios coincidentes.
En este caso no había respuestas correctas o incorrectas, sino que se buscaba saber cuál de los dos productos era el más gustado por los participantes. Según Farfán, 2009, la preferencia indica orden y no necesariamente que la muestra preferida sea la más aceptada, o que la menos preferida sea equivalente a rechazada.

\section{Resultados}

\section{Resultados de prueba de preferencia}

De acuerdo a la Figura 2, al 5\% de significancia, se debieron haber tenido al menos 24 juicios coincidentes para alguno de los dos productos evaluados para obtener una diferencia significativa.

Como se observa en la figura 3, solamente 20 personas optaron por el de aceituna y 15 optaron por el de alcaparra, sin obtenerse en ningún caso una diferencia significativa. 


\begin{tabular}{|c|c|c|c|c|c|c|}
\hline \multicolumn{7}{|c|}{ LA EVALUACIÓN SENSORIAL DE LOS ALIMENTOS } \\
\hline \multirow{3}{*}{$\begin{array}{l}\text { Número } \\
\text { de juicios }\end{array}$} & \multicolumn{3}{|c|}{ Pruebas de "Dos colas"* } & \multicolumn{3}{|c|}{ Pruebas de "Una cola"** } \\
\hline & \multicolumn{3}{|c|}{ Nivel de probabilidad } & \multicolumn{3}{|c|}{ Nivel de probabilidad } \\
\hline & $5 \%$ & $1 \%$ & $0.1 \%$ & $5 \%$ & $1 \%$ & $0.1 \%$ \\
\hline 31 & 22 & 24 & 25 & 21 & 23 & 25 \\
\hline 32 & 23 & 24 & 26 & 22 & 24 & 26 \\
\hline 33 & 23 & 25 & 27 & 22 & 24 & 26 \\
\hline 34 & 24 & 25 & 27 & 23 & 25 & 27 \\
\hline 35 & 24 & 26 & 28 & 23 & 25 & 27 \\
\hline 36 & 25 & 27 & 29 & 24 & 26 & 28 \\
\hline 37 & 25 & 27 & 29 & 24 & 27 & 29 \\
\hline 38 & 26 & 28 & 30 & 25 & 27 & 29 \\
\hline 39 & 27 & 28 & 31 & 26 & 28 & 30 \\
\hline 40 & 27 & 29 & 31 & 26 & 28 & 31 \\
\hline 41 & 28 & 30 & 32 & 27 & 29 & 31 \\
\hline 42 & 28 & 30 & 32 & 27 & 29 & 32 \\
\hline 43 & 29 & 31 & 33 & 28 & 30 & 32 \\
\hline 44 & 29 & 31 & 34 & 28 & 31 & 33 \\
\hline 45 & 30 & 32 & 34 & 29 & 31 & 34 \\
\hline 46 & 31 & 33 & 35 & 30 & 32 & 34 \\
\hline 47 & 31 & 33 & 36 & 30 & 32 & 35 \\
\hline 48 & 32 & 34 & 36 & 31 & 33 & 36 \\
\hline 49 & 32 & 34 & 37 & 31 & 34 & 36 \\
\hline 50 & 33 & 35 & 37 & 32 & 34 & 37 \\
\hline 60 & 39 & 41 & 44 & 37 & 40 & 43 \\
\hline 70 & 44 & 47 & 50 & 43 & 46 & 49 \\
\hline 80 & 50 & 52 & 56 & 48 & 51 & 55 \\
\hline
\end{tabular}

Figura 2. Tabla de significancia para pruebas de dos muestras (Anzaldúa-Morales, 1994).

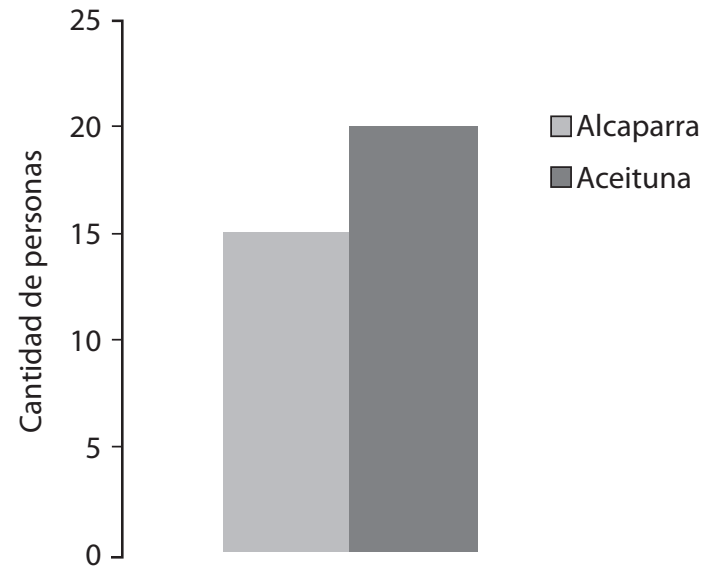

Figura 3. Cantidad de personas que prefirieron cada presentación de dip en la prueba de preferencia. Fuente: Autoras

\section{Discusión}

Como se describió en la metodología, del total de panelistas, se descartaron 4 debido a que no escogieron ningún dip tipo hummus. En un estudio de preferencia sobre potajes de frijol realizado por Leyva et al, 2010, también se tuvieron que eliminar 7 de 80 personas que no eligieron ni una ni otra de sus muestras.

En la figura 3 se observa que no se obtuvieron diferencias significativas entre las muestras analizadas del dip tipo hummus de aceituna y alcaparra $(p>0.05)$, lo cual significa que no hubo una tendencia definida hacia uno o hacia otro, sino que esto demuestra una aceptación hacia ambos productos. 
En otros estudios también se han encontrado estos resultados como en el realizado por Leyva et al, 2010, en donde encontraron que para 73 participantes no hubo diferencias significativas frente a la preferencia por potajes de frijol. Carrillo et al, 2011 tampoco encontraron diferencias significativas cuando evaluaron cuatro muestras de frijol nutricionalmente fortificado para población nicaragüense $(\mathrm{p}>0,05)$.

López, 2009, por el contrario, encontró diferencias significativas con un nivel de significancia del $5 \%$ entre dos tratamientos para chorizo con trehalosa, determinando que el tratamiento con mayor preferencia era el chorizo ahumado con $2 \%$ de trehalosa, ya que el mínimo de preferencia debía ser de 59 votos y se obtuvieron 63 votos. Mezquita et al, 2008, también encontraron diferencias significativas en una prueba de preferencia con dos formulaciones de bebidas de alto contenido proteico $(\mathrm{p}<0.05)$.

Como se muestra en la Figura 3, no se encontraron diferencias significativas entre cada tipo de hummus. Esto sugiere que se deben hacer aún más estudios para determinar si el comercializar ambos es factible o no.

De acuerdo a los comentarios de los participantes, la razón por la que escogieron el dip tipo hummus de aceituna fue por su sabor suave, por tener una cantidad ideal de aceituna y su textura, características que reflejan una formulación correcta. Esto se recomienda sea estudiado con más profundidad en una prueba de grado de satisfacción con una escala hedónica y en un focus group.

Con respecto al dip tipo hummus de alcaparra, la razón por la que fue escogido fue por su sabor a Gandul, menos grasoso y por ser un alimento que se presta a querer probarlo de nuevo. Según López, 2003, existen alimentos que generan este tipo de sensación, como el chile picante. Este producto, con el contacto con las neuronas sensoriales resultan en liberación de opioide, como las endorfinas, las cuales bloquean el dolor y provocan un estado placentero (como sucede con el chile picante, el cual genera la misma sensación adictiva).

Las razones por las que escogieron el de aceituna y no el de alcaparra fueron por ser este más suave, por tener una cantidad aceptable de ajo, por tener menos sal y por ser menos ácido que el de alcaparra. Todas estas características deben mejorarse antes de realizar otras pruebas con este dip, las cuales deberían ser, al igual que el de aceituna, de grado de satisfacción y un focus group.

\section{Conclusiones}

- No se encontraron diferencias significativas entre las pruebas de preferencia del dip tipo hummus sabor a alcachofa y el sabor a aceituna.

- Se encontraron comentarios favorables para cada uno de los dip tipo hummus.

- El dip tipo hummus sabor a alcachofa fue agradable para 15 panelistas por su sabor a Gandul, menos grasoso y por tener la característica de querer probarlo de nuevo.

- El dip tipo hummus sabor a aceituna fue agradable para 20 personas por ser más suave, por tener una cantidad aceptable de ajo, por tener una cantidad agrabable de sal y por ser menos ácido.

\section{Bibliografía}

ANDRÉS, C. 2013. Determinación de los descriptores sensoriales de un dip tipo hummus a base de la variedad criolla de Gandul (Cajanus Cajan (L.) Millsp.) mediante dos mini grupos focales. Por publicarse en "Cuadernosde Investigación, 5(1)".

ANZALDÚA-MORALES, A. 1994. La evaluación sensorial de los alimentos en la teoría y la práctica. Acribia, Zaragoza.

ARTAVIA ESTRADA, N. 2010. Efecto de la matriz alimentaria y el método de evaluación sobre el agrado por el dulzor. Universidad de Costa Rica. Facultad de ciencias agroalimentarias. Escuela de Tecnología de Alimentos. 96 p. (tesis).

BOSKOU, D. 2012. Mediterranean food products: research and development. En CIHEAM. (Ed.). MediTERRA. Francia. Recuperado de: http://www.cairn.info/publications-de-CIHEAM---46781.htm

CARRILLO, P; CHOW, Z; CUADRA, S; BRENES, D \& PACHÓN, H. 2011. Aceptación de tres líneas de frijol 
(Phaseous vulgaris L.) mejorado nutricionalmente por los consumidores nicaragüenses. Perspectivas en Nutrición Human. 13(2): 179-189. Recuperado de: http://revinut.udea.edu.co/index.php/nutricion/article/ viewArticle/179

CÉSPEDES, G. 2012. Director de la Asociación de productores de Gandul. Comunicación personal. La Tigra, San Carlos.

FARFÁN, E. 2009. Aprovechamiento del suero dulce de queso campesino para la elaboración de una bebida refrescante con sabor a mora. Universidad Nacional Abierta y a Distancia "UNAD" Facultad de Ciencias Básicas, Tecnología e Ingeniería Programa Ingeniería de Alimentos Cead Zipaquirá, Cundinamarca. 63 p. (tesis).

GONZÁLEZ, L. 2012. Gandul. Comunicación personal. San José, Costa Rica.

HERNÁNDEZ, E. 2005. Evaluación Sensorial. Universidad Nacional Abierta y a Distancia (UNAD) Bogotá Colombia. Recuperado de: INTA. 2009. Manejo de la seguridad alimentaria de las comunidades indígenas del Municipio de Waspam (GDCP/NIC/001/SPA): propuesta de modelos productivos. Recuperado de: https:// docs.google.com/viewer?a=v\&q=cache:9q14Kp6tN D8J:coin.fao.org/cms/media/13/13436731286680/ mejora_san_waspan.pdf $+\& h l=e s \& g l=c r \& p i d=b l \& s-$ rcid=ADGEESgYIugbBMEnc7Pgefv4AijysLXZK Y9-XEOCV8x4STPN0IW3qcQDoabMXjW7vnG2 xbRYPQ8ulaH6d2xByVMOZ7g3ERqTnIIHv0LS_ Yr1iI07Ktq25hdfsBkUsrOuwe-HinWtAGX3\&sig=A HIEtbT7jFVdVTxZNpkeZ6VCZzKLScKSwA

LEYVA-MARTÍNEZ, R; PACHÓN, H; CHAVECOPÉREZ, O; PERMUY- ABELEIRA, N; , FERRAZTELLEZ, Y; CABALLERO. 2010. Evaluación sensorial de frijol (Phaseolus vulgaris L.) mejorado nutricionalmente en dos comunidades cubanas. Agron. Mesoam. 21(2): 281-288. San Pedro. Recuperado de: http://www.scielo.sa.cr/scielo.php?script=sci_ arttext\&pid=S1659-13212010000200007

LÓPEZ, G. 2003. Chilli: la especia del nuevo mundo. Ciencias 69. Recuperado de: http://scholar.google. com/scholar?q=L\%C3\%93PEZ\%2C+G.+2003.+Chil $\mathrm{li} \% 3 \mathrm{~A}+\mathrm{la}+$ especia+del+nuevo+mundo\&btnG=\&hl= es\&as_sdt=0

LÓPEZ, L. 2009. Evaluación del efecto crioprotector de tres concentraciones de trehalosa sobre propiedades sensoriales y físicas de un chorizo ahumado. Zamorano: Escuela Agrícola Panamericana. 47 p. (tesis). Recuperado de: http://bdigital.zamorano.edu/ handle/11036/226

MAG. 1991. Gandul. Aspectos técnicos sobre cuarentena y cinco cultivos agrícolas de Costa Rica. Dirección general de Investigación y extensión agrícola. San José. Recuperado de: http://www.mag.go.cr/bibioteca_virtual_ciencia/tec-gandul.pdf

MEZQUITA, P; ACOSTA, E; ROJAS, G; ROMEROPALACIOS, N \& ARCOS, R. 2008. Desarrollo de una bebida de alto contenido proteico a partir de algarrobo, lupino y quinoa para la dieta de preescolares. Nutr. Hosp. 27 (1). Recuperado de: http://scielo.isciii.es/scielo.php?pid=S0212$16112012000100030 \&$ script=sci_arttext\&tlng=ptrg

ROBLEDO, L. s.f. Gandul. Recuperado de: http://es.scribd. com/doc/31118870/Cajanus-Cajan-Gandul

ROESSLER, E.B; BAKER, G.A \& AMERINE, M.A. 1956 .One-tailed and two tailed tests in organoleptic comparisons. Food Res. 21. 117 p. 